\title{
Electronic Structure of CePtIn and LaPtIn Compounds
}

\author{
A. Jezierski ${ }^{a}$, B. Penc $^{b}$, A. SzytuŁa ${ }^{b}$ And A. Winiarski ${ }^{c}$ \\ ${ }^{a}$ Institute of Molecular Physics, Polish Academy of Sciences, M. Smoluchowskiego 17, 60-179 Poznań, Poland \\ ${ }^{b}$ M. Smoluchowski Institute of Physics, Jagiellonian University, W.S. Reymonta 4, 30-059 Kraków, Poland \\ ${ }^{c}$ A. Chełkowski Institute of Physics, University of Silesia, Uniwersytecka 4, 40-007 Katowice, Poland
}

(Received January 24, 2012; in final form April 17, 2012)

\begin{abstract}
The electronic structure of the ternary RPtIn $(\mathrm{R}=\mathrm{La}, \mathrm{Ce})$ compounds, which crystallize in the hexagonal ZrNiAl-type structure, was studied by X-ray photoelectron spectroscopy measurements and calculation using the $a b$ initio methods (linear muffin-tin orbital in the atomic sphere approximation, full potential linear muffin-tin orbital, full potential linear orbital). The results showed that the valence band in these compounds is formed by the Pt $5 d$ and In $5 s$ and $5 p$ states. The band calculations with spin-orbit coupling have shown that the Ce $4 f$ peaks consist of two peaks above the Fermi level that correspond to the Ce $4 f_{7 / 2}$ and $4 f_{5 / 2}$ doublet and wide peaks corresponding to the La $4 f$ states. The analysis of Ce $3 d$ spectra on the basis of the Gunnarsson-Schönhammer model gives hybridization of $4 f$ orbitals with the conduction electron band equal to $170 \mathrm{meV}$.
\end{abstract}

PACS: 71.20.-b, 71.20.Lp, 71.27.+a, 79.60.-i

\section{Introduction}

The equiatomic ternary compounds CePtIn and LaPtIn crystallize in the hexagonal $\mathrm{ZrNiAl}$ structure (space group $P \overline{6} 2 m$ ) [1]. CePtIn is classified as dense Kondo material [2]. Heat capacity $\left(C_{p}\right)$ measurements of CePtIn yield $C_{p} / T \approx 1 \mathrm{~J} /\left(\mathrm{K}^{2} \mathrm{~mol}\right)$ at $60 \mathrm{mK}$ that indicates heavy fermion properties. The specific heat and electrical resistivity studies indicate the absence of magnetic order up to $50 \mathrm{mK}$ [3]. Temperature dependence of the electrical resistivity of LaPtIn indicates the metallic character and results for $\left(\mathrm{Ce}_{1-x} \mathrm{La}_{x}\right) \mathrm{PtIn}$ system indicate its change of the properties with increasing La content [4].

In the CeTIn compounds with increase in the unit cell volume the magnetic properties change from the Pauli paramagnet for CeNiIn to antiferromagnetical order found in CePdIn $\left(T_{\mathrm{N}}=1.8 \mathrm{~K}\right)$ and CeAuIn $\left(T_{\mathrm{N}}=\right.$ $5.7 \mathrm{~K}$ ). Simultaneously, a decrease in the Kondo temperature from $T_{\mathrm{K}}=94 \mathrm{~K}$ for CeNiIn to $3.3 \mathrm{~K}$ for CePdIn was reported [5]. The CePtIn compound with $T_{\mathrm{K}}=11 \mathrm{~K}$ and no magnetic order lie between those two border regions. Determination of the parameters of the electronic structure of this compound is interesting for explanation of the described changes in physical properties.

The photoemission method (PES) is considered to be the most successful way to study the nature of the Ce $4 f$ states [6]. The main aim of our investigations was to find correlations between the data on the electronic structure of the investigated compounds and hybridization of the $4 f$ level in the photoemission spectra of the valence band $(\mathrm{VB})$. In practice, the small cross-section of the $4 f$ states comparing to the $d$ ones makes the analyses very difficult when using classical photoemission. More reliable information about the $4 f$ states can be achieved using resonant photoemission. Also the Ce $3 d$ core-level photoemission lines have been widely used to diagnose hybridization strength of the $4 f$ levels in the Ce-based systems [6].

In this work we present the data concerning the electronic structure of RPtIn $(\mathrm{R}=\mathrm{La}, \mathrm{Ce})$ compounds supported by $a b$ initio calculations and PES results.

\section{Experimental details}

Polycrystalline samples of RPtIn ( $\mathrm{R}=\mathrm{La}$, Ce) compounds were obtained by arc melting of stoichiometric amounts of the elements (Merck AG: Ce and La of $99.9 \%$ purity, $\mathrm{Pd}$ and In of $99.99 \%$ purity) under a high-purity argon atmosphere on a water-cooled copper hearth, using a titanium-zirconium alloy as a getter. In order to ensure good homogeneity the buttons were turned over and remelted several times. The final weight losses were lower than $0.1 \%$. Subsequently the samples were annealed in evacuated quartz tubes at $800{ }^{\circ} \mathrm{C}$ for one week. The quality of the products was checked by X-ray powder diffraction using Philips PE-3710 apparatus $\left(\mathrm{Cu} K_{\alpha}\right.$ radiation) at room temperature. All of the reflections were indexed within the hexagonal structure of ZrNiAl-type. For processing the collected data the Rietveld-type program Fullprof was used [7]. The determined structure parameters for RPtIn $(\mathrm{R}=\mathrm{La}, \mathrm{Ce})$ compounds are in good agreement with the previous data [1]. 
The X-ray photoelectron spectra (XPS) were obtained at room temperature using a Multipurpose Electron Spectrometer PHI5700/660 with monochromatised radiation $\mathrm{Al} K_{\alpha}(h \nu=1486.6 \mathrm{eV})$ radiation source and the overall resolution of $0.3 \mathrm{eV}$. Binding energies were referred to the Fermi level $\left(E_{\mathrm{F}}=0\right)$. The spectrometer was calibrated using the $\mathrm{Cu} 2 p_{3 / 2}(932.5 \mathrm{eV}), \mathrm{Ag} 3 d_{5 / 2}$ $(368.1 \mathrm{eV})$ and $\mathrm{Au} 4 f_{7 / 2}(84.0 \mathrm{eV})$ core-level photoemission spectra. Surface of the samples was mechanically cleaned by scraping with a diamond file in a preparation chamber under high vacuum conditions $\left(10^{-9} \mathrm{mbar}\right)$. The Shirley method was used to subtract background; the experimental spectra obtained in this manner were numerically refined using the $80 \%$ Gaussian and $20 \%$ Lorentzian peak shape [8].

TABLE I

Structural parameters of CePtIn and LaPtIn at room temperature.

\begin{tabular}{c|c|c}
\hline \hline Compound & LaPtIn & CePtIn \\
\hline$a[\AA]$ & $7.823(1)$ & $7.706(1)$ \\
$c[\AA]$ & $4.165(1)$ & $4.097(1)$ \\
$a / c$ & $1.878(1)$ & $1.881(1)$ \\
$V\left[\AA^{3}\right]$ & $220.74(11)$ & $210.69(10)$ \\
R 3g $(x, 0,1 / 2)$ & $0.586(1)$ & $0.0 .588(1)$ \\
In 3f $(x, 0,0)$ & $0.249(1)$ & $0.251(1)$ \\
Pt1 1b $(0,0,1 / 2)$ & & \\
Pt2 2c $(1 / 3,2 / 3,0)$ & &
\end{tabular}

The electronic density of states (DOS) of RPtIn ( $\mathrm{R}=$ La, Ce) has been calculated by the spin-polarized self-consistent tight binding linear muffin-tin orbital (TB LMTO) method in the atomic sphere approximation (ASA) [9, 10] using the structural parameters from Table I. The scalar relativistic approximation for band electrons and the full relativistic treatment of frozen core electrons were applied. The exchange correlation potential was assumed in the form of von Barth and Hedin [11] and gradient corrections were also included [12].

\section{Results}

The calculated band structure of CePtIn is shown in Fig. 1. Above the Fermi level $(0-0.5 \mathrm{eV})$ the Ce $4 f$ state is observed. In the region of $3-4 \mathrm{eV}$ the $\mathrm{Pt} 5 d$ states is detected. On the basis of this data the density of states was calculated. The contribution from $\mathrm{Ce}$ or $\mathrm{La}, \mathrm{Pt}$ and $\mathrm{In}$ and the total density of states for CePtIn and LaPtIn calculated by scalar relativistic method are shown in Fig. 2.

Both methods of calculation give nearly similar DOS. Above the Fermi level $\left(E_{\mathrm{F}}=0 \mathrm{eV}\right)$ the doublet corresponding to the Ce $4 f_{3 / 2}$ and $\mathrm{Ce} 4 f_{5 / 2}$ is observed. In the $3-4.5 \mathrm{eV}$ regions the states of $\mathrm{Pt} 5 d$ are detected. At $6 \mathrm{eV}$ the peaks of In $5 s$ are observed whereas In $5 p$ states form a band between 0 and $4.5 \mathrm{eV}$. Similar DOS is observed for LaPtIn however the La $4 f$ states form the wide peak at $2.5 \mathrm{eV}$ above the Fermi level.

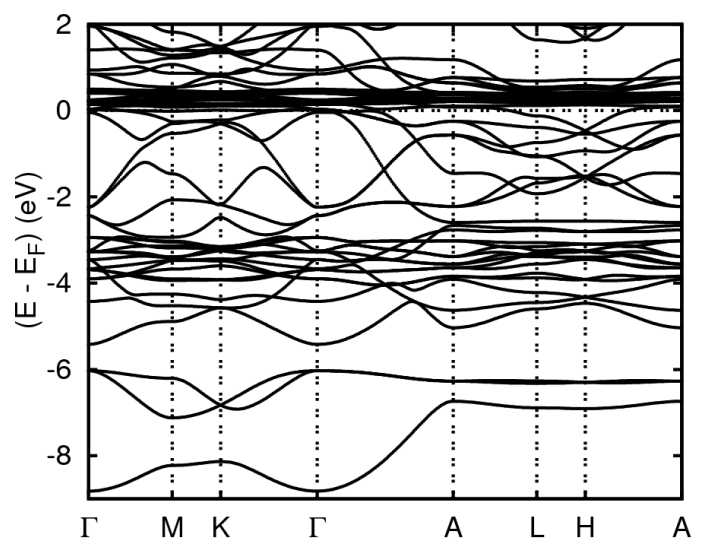

Fig. 1. Band structure of CePtIn.

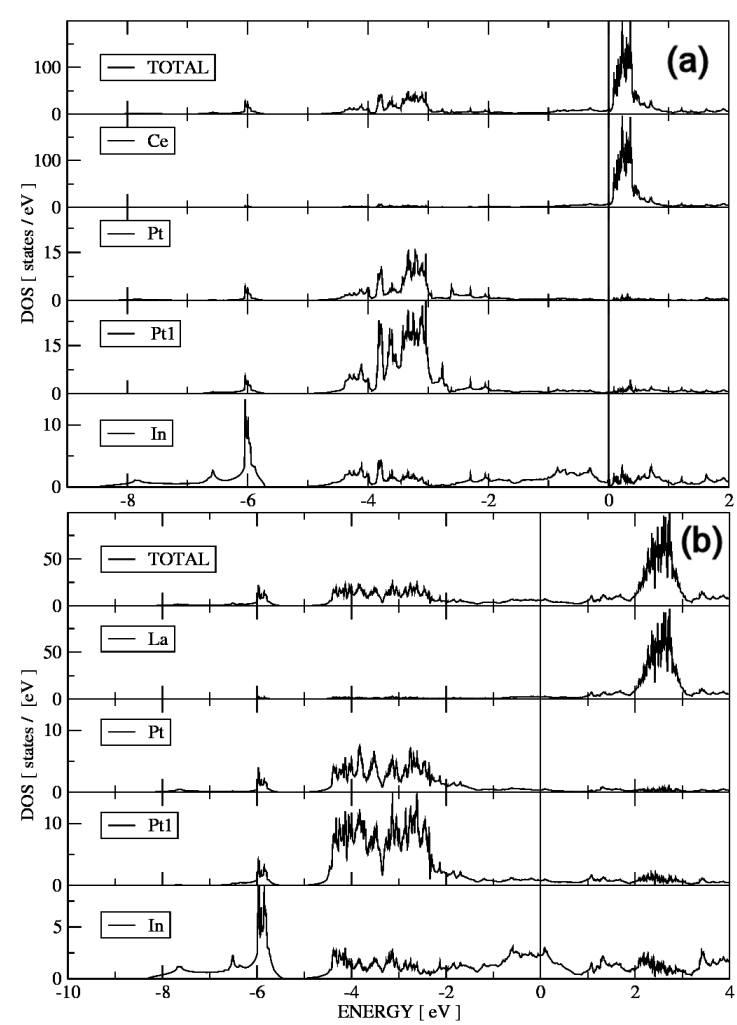

Fig. 2. Total density of states and the contribution from $\mathrm{Ce}$ or $\mathrm{La}, \mathrm{Pt}$ and In to the total density of states for CePtIn (a) and LaPtIn (b) calculated in the scalar-relativistic approximation.

TABLE II

Atomic concentration in RPtIn $(\mathrm{R}=\mathrm{La}, \mathrm{Ce})$ compounds.

\begin{tabular}{c|c|c|c}
\hline \hline Compound & $\mathrm{R} 3 d_{5 / 2}$ & $\mathrm{Pt} 4 f_{7 / 2}$ & In $3 d_{5 / 2}$ \\
\hline CePtIn & 30.70 & 33.4 & 35.90 \\
LaPtIn & 34.03 & 32.12 & 33.85
\end{tabular}


The calculated values of the density of states at the Fermi level $N\left(E_{\mathrm{F}}\right)$ for both methods of calculation are shown in Table II.

\section{TABLE III}

Density of states at the Fermi level $N\left(E_{\mathrm{F}}\right)$ for both methods of calculations.

\begin{tabular}{c|c|c}
\hline \hline & Scalar realistic & Full relativistic \\
\hline LaPtIn & 169.9 & 157.8 states \\
CePtIn & 313.2 & 350.1 states
\end{tabular}

These results indicate that the increase in $N\left(E_{\mathrm{F}}\right)$ for CePtIn is caused by the Ce $4 f$ states. The calculated occupation of the Ce $4 f$ level is equal to 0.97 .

Figure 3 shows the XPS spectra of CePtIn and LaPtIn collected in a wide $0-1400 \mathrm{eV}$ binding energy range, the binding energies are related to the Fermi level $\left(E_{\mathrm{F}}=\right.$ $0 \mathrm{eV}$ ). The results of the analysis of the intensity of the selected peaks corresponding to $\mathrm{R} 3 d_{5 / 2}, \mathrm{Pt} 4 f_{7 / 2}$ and In $3 d_{5 / 2}$ states are given in Table III. The obtained results indicate that in both compounds the ratio 1:1:1 is retained. In general, the most important data for CePtIn were obtained from three regions: VB (binding energy 0-10 eV), Ce $4 d$ states $(100-120 \mathrm{eV})$ and Ce $3 d$ states (880-930 eV). The XPS VB for both compounds is presented in Fig. 4. These spectra present mainly the dominant contribution of the Pt states. For CePtIn this corresponds to two peaks: at 3.2 and $4.5 \mathrm{~K}$. Similar two peaks at 3.2 and $4.4 \mathrm{eV}$ are observed in LaPtIn. The adequate values of spin-orbit splitting are equal to 2.5 and $1.2 \mathrm{eV}$.

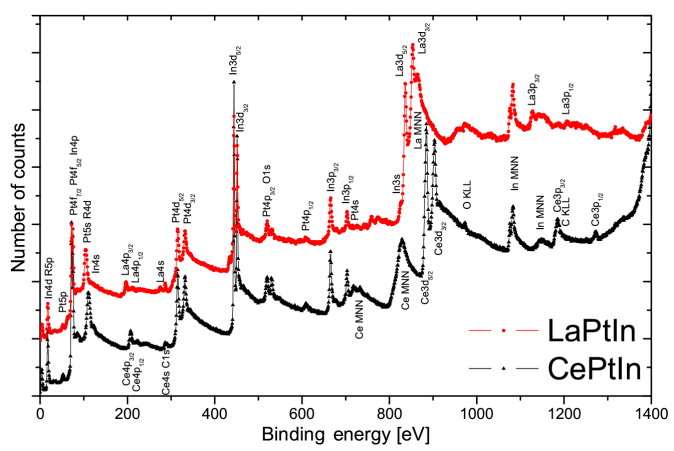

Fig. 3. XPS Al $K_{\alpha}$ spectra of LaPtIn and CePtIn collected at room temperature within $0-1400 \mathrm{eV}$ binding energy range ( $\mathrm{Al} K_{\alpha}$ radiation).

A broad peak at $6.1 \mathrm{eV}$ for CePtIn and at $6.6 \mathrm{eV}$ for LaPtIn corresponds to In $5 s$ states. Near the Fermi level two peaks at 0.5 and $2.1 \mathrm{eV}$ for CePtIn and one at 0.5 and $1.9 \mathrm{eV}$ for LaPtIn were observed. The peaks at $0.5 \mathrm{eV}$ are connected to the $5 d 6 s^{2}$ state where the peak at $1.9 \mathrm{eV}$ for LaPtIn is connected with the In $5 p$ state. For CePtIn the peak at $2.1 \mathrm{eV}$ is probably connected with the Ce $4 f$ and In $5 p$ state.

At about $110 \mathrm{eV}$ a broad peak of the Ce $4 f$ states can be noticed (see Fig. 5). The Ce $4 d_{5 / 2}$ lines is decomposed into four peaks with the binding energies equal to

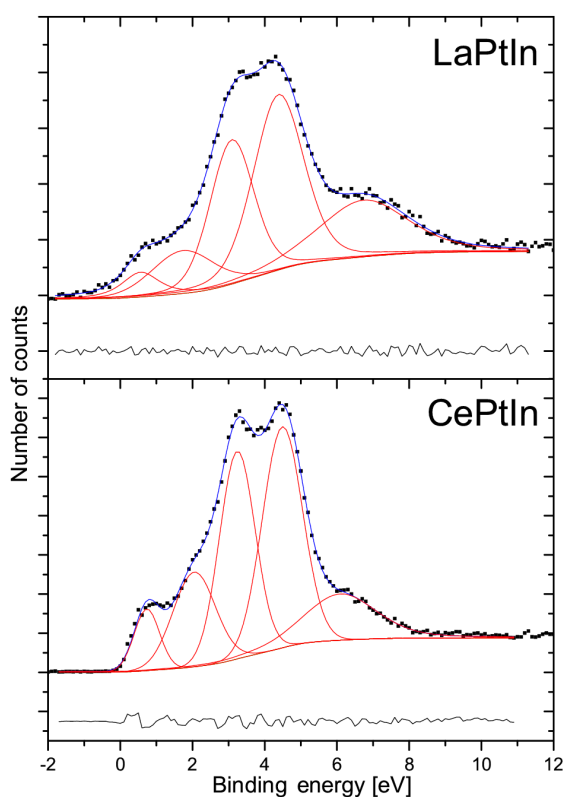

Fig. 4. Deconvoluted photoemission valence band spectra for LaPtIn and CePtIn.

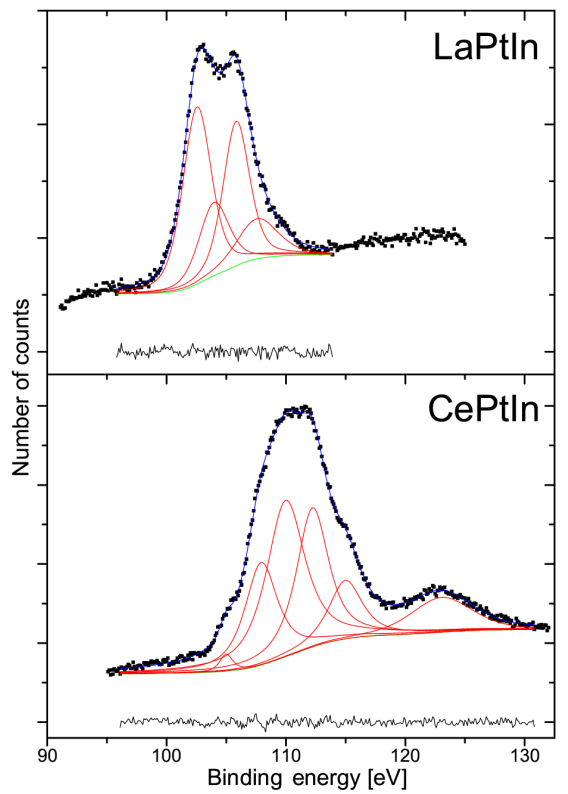

Fig. 5. Deconvoluted Ce $4 d$ spectrum in CePtIn and La $4 d$ spectrum in LaPtIn.

$107.9,110.0,112.2$, and $115.0 \mathrm{eV}$. Unfortunately analysis of the structure of this peak is possible. In LaPtIn in this region two peaks corresponding to the La $4 d_{5 / 2}$ and $\mathrm{La} 4 d_{3 / 2}$ are observed at 102.55 and $105.85 \mathrm{eV}$ and the spin-orbit splitting is equal to $\Delta=3.3 \mathrm{eV}$. These data are in good agreement with the data for the pure elements 102.5, 105.3, and $2.8 \mathrm{eV}$ [13].

Figure 6 illustrates the Ce $3 d$ XPS spectrum of the CePtIn compound. Two lines corresponding to the spin- 
orbit splitting equal to $18.4 \mathrm{eV}\left(3 d_{3 / 2}\right.$ and $3 d_{5 / 2}$ doublets) are observed. Additionally, each line consists of two sublines corresponding to the $3 d^{9} 4 f^{1}$ and $3 d^{8} 4 f^{2}$ states. The analysis of these components was based on the DoniachŠunjić theory [14]. Within this approach the intensity ratio $r_{1}=I\left(f^{2}\right) /\left[I\left(f^{2}\right)+I\left(f^{1}\right)\right]$ can be calculated $\left(I\left(f^{n}\right)\right.$ is the integral intensity of relevant components). From $r_{1}$ value it is possible to determine the coupling parameter $\Delta$ basing on the calculations of the intensity ratio $r_{1}$ as a function of $\Delta$ previously published [6]. The coupling parameter $\Delta$ is defined as $\pi V^{2} \rho_{\max }$, where $V$ is the hybridization and $\rho_{\max }$ is the maximum of the density of conduction electron states. The value of the intensity ratio $r_{1}$ is equal to 0.33 which corresponds to the $\Delta$ parameter of $170 \mathrm{eV}$.

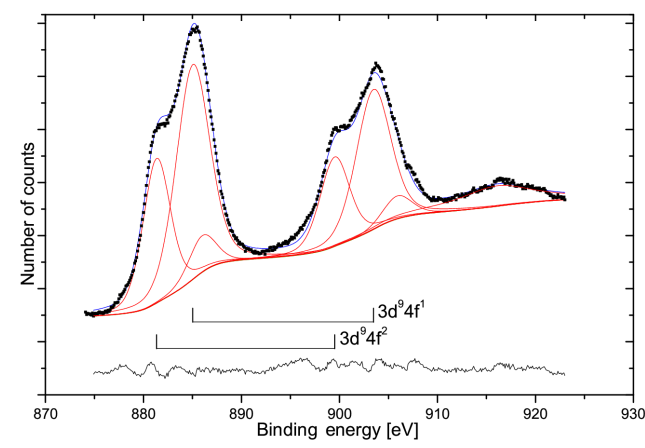

Fig. 6. Deconvoluted X-ray photoelectron spectra of Ce $3 d_{5 / 2}$ and Ce $3 d_{3 / 2}$ electron states in CePtIn.

\section{TABLE IV}

The binding energies and the spin-orbit splitting $\Delta_{\mathrm{S}-\mathrm{O}}$ (in $\mathrm{eV}$ ) in RPtIn ( $\mathrm{R}=\mathrm{La}, \mathrm{Ce})$ compounds, compared with the data for pure element.

\begin{tabular}{c|c|c|c}
\hline \hline & LaPtIn & CePtIn & $\begin{array}{c}\text { Pure element } \\
{[13]}\end{array}$ \\
\hline In $4 d_{5 / 2}$ & 16.8 & 16.85 & 16.6 \\
$4 d_{3 / 2}$ & 17.7 & 17.81 & 17.4 \\
$\Delta_{\mathrm{S}-\mathrm{O}}$ & 0.9 & 1.0 & 0.8 \\
$\mathrm{Pt} 4 f_{7 / 2}$ & 71.6 & 71.6 & 71.1 \\
$4 f_{5 / 2}$ & 74.9 & 74.9 & 74.7 \\
$\Delta_{\mathrm{S}-\mathrm{O}}$ & 3.3 & 3.3 & 3.6 \\
$\mathrm{Pt} 4 d_{5 / 2}$ & 314.8 & 314.8 & 314.6 \\
$4 d_{3 / 2}$ & 331.6 & 331.6 & 331.6 \\
$\Delta_{\mathrm{S}-\mathrm{O}}$ & 16.8 & 16.8 & 17.0 \\
$\mathrm{In} 3 d_{5 / 2}$ & 443.7 & 443.8 & 443.9 \\
$3 d_{3 / 2}$ & 451.3 & 451.3 & 451.4 \\
$\Delta_{\mathrm{S}-\mathrm{O}}$ & 7.6 & 7.5 & 7.5
\end{tabular}

The evidence of the intermediate valence of Ce ion is not clear because the $\mathrm{Ce} 3 d^{9} 4 f^{0}$ peak at $916.2 \mathrm{eV}$ and the Ce $4 d$ one connected with the $f^{0}$ final state near $122.5 \mathrm{eV}$ are broad.
In the next step the positions of the In $4 d_{1 / 2}$ and $4 d_{3 / 2}$ and $3 d_{5 / 2}$ and $3 d_{3 / 2}$ and $\mathrm{Pt} 4 f_{7 / 2}$ and $4 f_{5 / 2}$ and $4 d_{5 / 2}$ and $4 d_{3 / 2}$ core levels were analyzed. It was shown that the values of the energy and spin-orbit splitting $\Delta_{\mathrm{S}-\mathrm{O}}$ are similar for both compounds and do not change in comparison with the table values in Ref. [13] (see Table IV).

\section{Summary}

In this work we investigated the electronic structure of CePtIn and LaPtIn. The small difference in the valence band of these compounds was observed. From the $3 d$ spectrum the hybridization parameter equal to $170 \mathrm{eV}$ was determined which is close to these value reported for the isostructural CePdIn $(130 \mathrm{eV})$ [5], in spite that these compounds have different properties. The presented data do not give explanation of anomalous properties of CePtIn. The Ce $3 d$ and Ce $4 d$ spectra do not give any evidence of intermediate valence of $\mathrm{Ce}^{3+}$ in CePtIn.

\section{Acknowledgments}

This work was partially supported by Ministry of Science and Higher Education in Poland under Grant no. N N202 201039.

\section{References}

[1] R. Ferro, R. Marazza, G. Rambaldi, Z. Metallkd. 65, 40 (1974).

[2] K. Satoh, T. Fujita, Y. Maeno, Y. Uwatoko, H. Fujii, J. Phys. Soc. Jpn. 56, 692 (1990).

[3] Y. Maeno, M. Takahashi, T. Fujita, Y. Maeno, Y. Uwakoto, H. Fujii, T. Okamoto, Jpn. J. Appl. Phys. 26 (suppl. 3), 545 (1987).

[4] F.C. Ragel, P de V. du Plessis, A.M. Strydom, J. Phys., Condens. Matter 21, 046008 (2009).

[5] A. Szytuła, B. Penc, Ł. Gondek, Acta Phys. Pol. A 111, 475 (2007) and references therein.

[6] J.C. Fuggel, F.U. Hillebrecht, Z. Żołnierek, R. Lässer, Ch. Frieburg, O. Gunnersson, K. Schönhammer, Phys. Rev. B 27, 7330 (1983).

[7] J. Rodriguez-Carvajal, Physica B 192, 55 (1993).

[8] D.A. Shirley, Phys. Rev. B 5, 4709 (1975).

[9] O.K. Andersen, O. Jepsen, M. Sob, in: Electronic Structure and Its Applications, Ed. M. Yussanff, Springer-Verlag, Berlin 1987, p. 2.

[10] O.K. Andersen, O. Jepsen, Phys. Rev. Lett. 53, 2571 (1984).

[11] U. von Barth, L. Hedin, J. Phys. C 5, 1629 (1972).

[12] D. Hu, D.C. Langreth, Phys. Scr. 32, 91 (1985).

[13] S. Hüfner, in: Photoemission Spectroscopy, Springer-Verlag, Berlin 1994, p. 453.

[14] S. Doniach, M. Šunjić, J. Phys. C 3, 285 (1970). 